Pacific

Journal of

Mathematics

ON THE TQFT REPRESENTATIONS OF THE MAPPING CLASS GROUPS

LOUIS FUNAR 


\title{
ON THE TQFT REPRESENTATIONS OF THE MAPPING CLASS GROUPS
}

\author{
LOUIS FUNAR
}

\begin{abstract}
We prove that the image of the mapping class group by the representations arising in the $S U(2)-T Q F T$ is infinite, provided that the genus $g \geq 2$ and the level of the theory $r \neq 2,3,4,6$ (and $r \neq 10$ for $g=2$ ). In particular it follows that the quotient groups $\mathcal{M}_{g} / N\left(t^{r}\right)$ by the normalizer of the $r$-th power of a Dehn twist $t$ are infinite if $g \geq 3$ and $r \neq 2,3,4,6,8,12$.
\end{abstract}

\section{Introduction.}

Witten [50] constructed a TQFT in dimension 3 using path integrals and afterwards several rigorous constructions arose, like those using the quantum group approach $([\mathbf{3 9}, \mathbf{2 5}])$, the Temperley-Lieb algebra $([\mathbf{3 0}, \mathbf{3 1}])$, the theory based on the Kauffman bracket $([4,5])$ or that obtained from the mapping class group representations and the conformal field theory $([\mathbf{2 7}])$.

Any TQFT gives rise to a tower of representations of the mapping class groups $\mathcal{M}_{g}$ in all genera $g$ and this tower determines in fact the theory, up to the choice of the vacuum vector (see $[\mathbf{1 0}, \mathbf{5}, \mathbf{4 8}]$ ). The aim of this paper is to answer whether the image of the mapping class groups is finite or not under such representations.

There is some evidence supporting the finiteness of this image group. First, in the Abelian $U(1)$-theory the representations can be identified with the monodromy of a system of theta functions. The latter is explicitly computed (see e.g. $[\mathbf{1 1}, \mathbf{1 5}]$ ) and it is easy to see that it factors through a finite extension (due to the projective ambiguity) of $S p(2 g, \mathbf{Z} / r \mathbf{Z})$, where $r$ is the level of the theory. For a general Lie group $G$, the monodromy associated to the genus 1 surfaces may also be determined (see $[\mathbf{1 8}, \mathbf{1 1}]$ ) using some formulas of Kac (see $[\mathbf{2 1}, \mathbf{2 2}]$ ) and again it factors through a finite extension of $S p(2 g, \mathbf{Z} / r \mathbf{Z})$, where $r$ is now the shifted level. This has already been suggested by the fact that the Reshetikhin-Turaev invariant for lens spaces $L_{a, b}$ ( $a \leq b$ running over the positive integers) takes only a finite number of distinct values, namely for the cosets mod $r$ of $a$ and $b$ (see for instance [29]). For low levels $r=4,6$ the whole tower of representations was described by Blanchet and Masbaum in $[\mathbf{6}]$ and by Wright $([\mathbf{5 1}, \mathbf{5 2}])$ and in particular the images are finite groups. On the other hand all TQFTs are associated 
to conformal field theories (abb. CFT) in dimension 2 (see e.g. [10, 50]) and the finiteness question appeared also in the context of classification of rational conformal field theories. For instance in [36] one asks whether the algebraic CFT have finite monodromy (which is equivalent to our problem for some classes of TQFTs, as for example the $S U(2)$-TQFT). Some of the irreducible representations of $S L(2, \mathbf{Z} / r \mathbf{Z})$ which admit extensions as monodromies of some CFT in all genera were discussed in $[\mathbf{8}, \mathbf{9}]$. Also in [24] the action of $S L(2, \mathbf{Z})$ on the conformal blocks was computed for all quantum doubles, and it could be proved that the image is always finite. Gilmer obtained in another way (see [14]) the finiteness of the image for $g=1$, in the $S U(2)$ theory, result which seems to be known in the conformal field theory community, and noticed also by M. Kontsevich. Meantime Stanev and Todorov [41] have a partial answer to this question in the case of the 4-punctured sphere, as we will explain below.

This is the motivation for our main result:

Theorem 1.1. The image $\rho\left(\mathcal{M}_{g}\right)$ of the mapping class group $\mathcal{M}_{g}$ under the representation $\rho$ arising in the $S U(2)-T Q F T$ (in both the BHMV and $R T$ versions) and respectively $S O(3)-T Q F T$ is infinite provided that $g \geq 2$, $r \neq 2,3,4,6$, and if $g=2$ also $r \neq 10$.

Let us mention that $\rho$ is only a projective representation and thus its image is well defined up to scalar multiplication by roots of unity of order $4 r$. To explain briefly what means the two versions BHMV and RT we recall that the $S U(2)$-TQFT was constructed either using the Kauffman bracket - and this is the BHMV version from [5] - or else using the coloured Jones polynomial - and this is the RT version from $[39,25]$. The invariants obtained for closed 3-manifolds are "almost" the same, but their TQFT extensions are different. That is the reason for considering here both of them, though as it should be very unlikely that the mapping class group representations do not share the same properties, in the two related cases.

Before we proceed let us outline the relationship with the results from [41], where the Schwarz problem is considered for the $\widehat{s u(2)}$ KnizhnikZamolodchikov equation. The authors determined whether the image of the mapping class group of the 4-punctured sphere is finite, thereby solving a particular case of our problem, however in a slightly different context. It would remain to identify the following two representations of the mapping class groups (in arbitrary genus):

- one is that arising from the conformal field theory based on the $\widehat{s u(2)}$ Knizhnik-Zamolodchikov equation. Tsuchiya, Ueno and Yamada [43] constructed the CFT using tools from algebraic geometry, for all Riemann surfaces.

- the other one is that arising in the RT-version of the $S U(2)-T Q F T$. 
There are some naturally induced representations of braid groups in both approaches, which can be proved to be the same by the explicit computations of Tsuchyia and Kanie [42].

Presumably the two representations of the mapping class groups are also equivalent, but a complete proof of this fact does not exist, on author's knowledge. First it should be established that the CFT extends to a TQFT in 3 dimensions, which is equivalent to control the behaviour of conformal blocks sheaves over the compactification divisor on the moduli space of curves. Observe that a different and direct construction of the associated TQFT can be given $([\mathbf{2 7}, \mathbf{2 8}, \mathbf{1 2}])$ if we assume the CFT has all the properties claimed by the physicists. Notice that a complete solution of that problem would furnish an entirely geometric description of the TQFT following Witten's prescriptions, in which the mapping class group representation is the monodromy of a projectively flat connection on some vector bundle of non-abelian theta functions over the Teichmuller space.

Thus we cannot deduce directly from [41] the finiteness of the mapping class group representation without assuming the previous unproved claim. Our purpose is to use instead the BHMV approach which has a simple and firmly established construction. We consider braid group representations using basically the monodromy of the holed spheres. The data we obtain is similar to that obtained by all the other means, hence also to that from $[41,42]$. Specifically, the idea of the proof of the main theorem is to identify a certain subspace of the space on which $\mathcal{M}_{g}$ acts, which is invariant to the action of a subgroup of $\mathcal{M}_{g}$, the last being a quotient of a pure braid group $P_{n}, n \geq 3$. Next we observe that the action of $P_{n}$ extends naturally to an action of the whole braid group $B_{n}$, and this it turns to factor through the Hecke algebra $H_{n}(q)$ of type $A_{n-1}$ at a root of unity $q$. This was inspired by the computations done by Tsuchyia and Kanie ([42], see also [41]) of the monodromy in the conformal field theory on $\mathbf{P}^{1}$. Now an easy modification of the Jones theorem $([20])$, about the generic infiniteness of the image of $B_{n}$ in Hecke algebra representations, will settle our question.

For fixing the notations, we denote by $r$ the level, which is supposed to be in this sequel the order of the roots of unity which appear in the definition of the invariants for the RT-version and respectively a quarter (or half) of it for the BHMV-version for $S U(2)$ (respectively $S O(3)$ ).

The groups $\mathcal{M}_{g} / N\left(t^{r}\right)$, quotients of $\mathcal{M}_{g}$ by the normalizer of a power of a Dehn twist, were previously considered for $r=2,3$ by Humphries in [17], and it was shown that these are finite groups for $r=2$ and arbitrary $g$, and infinite for $g=2$ and $r \geq 3$. This solved the problem 28 asked by Birman in [2], p. 219. We derive a generalization of that, to all other genera $g$, namely:

Corollary 1.2. The quotient groups $\mathcal{M}_{g} / N\left(t^{r}\right)$ are infinite for $g \geq 3, r \neq$ $2,3,4,6,8,12$. 
Proof. It is well-known (see [35], p. 379) that the image of a Dehn twist $\rho(t)$, in some nice basis, is a diagonal matrix whose entries are $(-1)^{j} A^{j^{2}+2 j}$, where $A$ is a $2 r$-th root of unity. It follows that, for odd $r, \rho(t)^{r}$, and for even $r, \rho(t)^{2 r}$ respectively, is a scalar matrix in this particular basis, and furthermore it is a scalar matrix in any other basis. Therefore, the image group $\rho\left(\mathcal{M}_{g}\right)$ (modulo multiplication by roots of unity of order $4 r$ ) is a quotient of $\mathcal{M}_{g} / N\left(t^{r}\right)$ (and respectively $\mathcal{M}_{g} / N\left(t^{2 r}\right)$ ), and now the claim follows.

Notice that the proof given by Humphries used the Jones representation [19] of $\mathcal{M}_{2}$ which arises as follows: The group $\mathcal{M}_{2}$ is viewed as a quotient of the braid group $B_{6}$, and some Hecke algebra representation factors throughout $\mathcal{M}_{2}$. For $g>2$ it is only a proper subgroup of $\mathcal{M}_{g}$ which is a quotient of $B_{2 g+2}$, so that an extension of the Jones representation to higher genus is not obvious.

It seems that not only the representations have infinite image, but the set of values the $S U(2)$-invariant (at a given level $r$ ) takes on the closed 3 -manifolds of fixed Heegaard genus $g$, is also infinite. Our result does not imply this stronger statement, because the infinite image we found comes from a subgroup of $K \subset \mathcal{M}(F)$ of homeomorphisms of the surface extending to the handlebody. In fact when we twist the gluing map of a Heegaard splitting by an element of $K$ we obtain a manifold homeomorphic to the former one. However it is very likely that the same method could be refined to yield this stronger statement.

We think that our theorem holds also for the case $g=2, r=10$ and the corollary is true more generally for $g \geq 3, r \geq 3$. The same ideas can be used for the $S U(N)$-TQFT to show that, in general, the corresponding representations of $\mathcal{M}_{g}$ have infinite images.

Acknowledgements. This work was done during author's visit at Columbia University, whose hospitality is gratefully acknowledged. We are thankful to Roland Bacher, Joan Birman, Christian Blanchet, Razvan Gelca, Patrick Gilmer, Gregor Masbaum, Jerome Los, Vlad Sergiescu, Gretchen Wright for their suggestions and comments and to the referee for pointing out some errors in the previous version and simplifying the proof.

\section{Preliminaries.}

\subsection{Hecke algebras and Temperley-Lieb algebras.}

We will outline briefly, for the sake of completeness, some basic notions concerning the Hecke algebras (see [49] for more details). Recall that the Hecke algebra of type $A_{n-1}$ is the algebra over $\mathbf{C}$ generated by $1, g_{1}, \ldots, g_{n-1}$ and the following relations:

$$
g_{i} g_{i+1} g_{i}=g_{i+1} g_{i} g_{i+1}, i=1,2, \ldots, n-2,
$$




$$
\begin{gathered}
g_{i} g_{j}=g_{j} g_{i},|i-j|>1, \\
g_{i}^{2}=(q-1) g_{i}+q, i=1,2, \ldots, n-1,
\end{gathered}
$$

where $q \in \mathbf{C}-\{0\}$ is a complex parameter. Denote this algebra by $H_{n}(q)$. It is known (see e.g. [7], p. 54-55) that $H_{n}(q)$ is isomorphic to the group algebra $\mathbf{C} S_{n}$ of the symmetric group $S_{n}$, provided that $q$ is not a root of unity.

Notice that $H_{n}(q)$ is the quotient of the group algebra $C B_{n}$ of the braid group $B_{n}$. The braid group is usually presented as generated by $g_{1}, \ldots, g_{n-1}$, together with the first two relations from above. In particular there is a natural representation of $B_{n}$ in $H_{n}(q)$.

From the quadratic relation satisfied by $g_{i}$ it follows that $g_{i}$ has at most two spectral values. For $q \neq-1$ set $f_{i}$ for the spectral projection corresponding to the eigenvalue -1 ; then $g_{i}=q-(1+q) f_{i}$, and another presentation of $H_{n}(q)$ can be obtained in terms of the generators $1, f_{1}, \ldots, f_{n-1}$, as follows:

$$
\begin{gathered}
f_{i} f_{i+1} f_{i}-q(1+q)^{-2} f_{i}=f_{i+1} f_{i} f_{i+1}-q(1+q)^{-2} f_{i+1}, i=1,2, \ldots, n-2, \\
f_{i} f_{j}=f_{j} f_{i},|i-j|>1, \\
f_{i}^{2}=f_{i}, i=1,2, \ldots, n-1 .
\end{gathered}
$$

The irreducible representations of Hecke algebras are well-known in the case when $H_{n}(q)$ are semi-simple, which means that $q$ is not a root of unity. The structure of $H_{n}(q)$ at roots of unity is more complicated (see for instance [49]) and as we will be concerned with this situation precisely we introduce also some smaller quotients (after $[\mathbf{1 6}, \mathbf{2 0}]$ ), namely the Temperley-Lieb algebras.

The algebras $A_{\beta, n}$ (following the convention from [16], Section 2.8) are generated over $\mathbf{C}$ by $1, e_{1}, \ldots, e_{n-1}$ and the relations:

$$
\begin{gathered}
e_{i} e_{i+1} e_{i}=e_{i} e_{i-1} e_{i}=\beta^{-1} e_{i}, i=1,2, \ldots, n-2, \\
e_{i} e_{j}=e_{j} e_{i},|i-j|>1, \\
e_{i}^{2}=e_{i}, i=1,2, \ldots, n-1 .
\end{gathered}
$$

Remark that $A_{\beta, n}$ is a quotient of the Hecke algebra $H_{n}(q)$, for $\beta=2+q+$ $q^{-1}$. In fact, the image of the projector $f_{i}$ is $1-e_{i}$. If we replace $e_{i}=1-f_{i}$ we find a presentation of $A_{\beta, n}$ as the quotient of $H_{n}(q)$ by adding the set of relations (see also Prop. 2.11.1 from [16], p. 123):

$$
g_{i} g_{i+1} g_{i}+g_{i} g_{i+1}+g_{i+1} g_{i}+g_{i}+g_{i+1}+1=0, \text { for } i=1, n-1 .
$$

It is known that the algebra $A_{\beta, 3}$ is semi-simple for $\beta \neq 1$ (or equivalently, $r \neq 3$ ) and $A_{\beta, 4}$ is semi-simple for $\beta \neq 1,2$ (equivalently $r \neq 3,4$ ). Moreover, 
when semi-simple these are multi-matrix algebras: $A_{\beta, 3}=M_{2}(\mathbf{C}) \oplus \mathbf{C}$, and $A_{\beta, 4}=M_{3}(\mathbf{C}) \oplus M_{2}(\mathbf{C}) \oplus \mathbf{C}$, (see Theorem 2.8.5, p. 98 from [16]).

\subsection{Mapping class group representations.}

Most of the material presented here comes from $[30,40,34]$. Let $A$ be a fixed complex number and $M$ be a compact oriented 3-manifold. The skein module $S(M)$ is the vector space generated by the isotopy classes (rel $\partial M$ ) of framed links, quotiented by the (skein) relations from Figure 1.

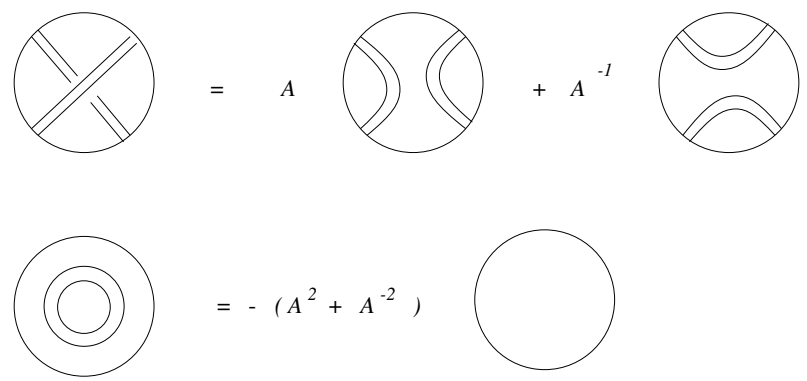

Figure 1. Skein relations.

For example $S\left(S^{3}\right)$ is one dimensional (as a module over $\mathbf{Z}\left[A, A^{-1}\right]$ ), with basis the empty link; the image of the framed link $L \subset S^{3}$ in $S\left(S^{3}\right)$ is the value of the Kauffman bracket evaluated at $A$. Notice that, in order to construct the TQFT we must specialize $A$ to be a primitive $2 r$-th root of unity. For even $r$ we obtain the $S U(2)$-TQFT (level $\frac{r}{2}$ with our convention) and for odd $r$ we obtain the $S O(3)$-TQFT (of level $r$ this time).

The skein space for the 3-ball with $2 n$ boundary (framed) points has an algebra structure, by representing the framed link in a planar projection sitting into a rectangle, and separating the points into two groups of $n$ on opposite sides. The multiplication is given by the juxtaposition of diagrams, and the algebra thus obtained can be identified with the the Temperley-Lieb algebra $A_{\beta, n}$, for a suitable $\beta$. The generators for $A_{\beta, n}$ are the elements $1_{n}, e_{1}, \ldots, e_{n-1}$ pictured in Figure 2.

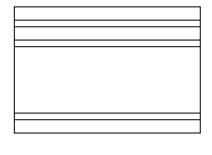

$1_{\mathrm{n}}$

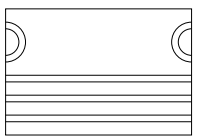

$\mathrm{e}_{1}$

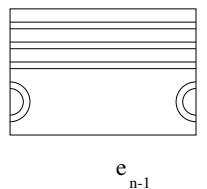

$e_{n-1}$

Figure 2. Generators of the Temperley-Lieb algebra.

Now the Jones-Wenzl idempotents $f^{(n)} \in A_{\beta, n}$ are uniquely determined by the conditions $f^{(n) 2}=f^{(n)}, f^{(n)} e_{i}=e_{i} f^{(n)}=0$, for $i=1,2, \ldots, n-1$, whenever $A$ is such that all $\Delta_{i}=(-1)^{i} \frac{A^{2 i+1}-A^{-2 i-1}}{A^{2}-A^{-2}}$ for $i=0,1, \ldots, n-1$ are non-zero. This implies that $f^{(n)} x=x f^{(n)}=\lambda_{x} f^{(n)}$, for all $x$, with a suitable chosen complex number $\lambda_{x}$. 
Denote in a planar diagram by a line labeled with $n$ (in a small rectangle) the element $1_{n} \in A_{\beta, n}$, and by a line with a dash labeled $n$ the insertion of the element $f^{(n)} \in A_{\beta, n}$. This will give a convenient description for the elements of skein modules.

One construction for the $S U(2)$-invariants via skein modules, was given in $[30,31]$ and latter extended to a TQFT in [5], and to higher $S U(N)$ invariants recently in [32].

Let us outline first the construction of the conformal blocks, which are the vector spaces associated to surfaces via the TQFT. Decompose the sphere $S^{3}$ as the union of two handlebodies $H$ of genus $g$, and $H^{\prime}$ with a small cylinder $F \times I$ over the surface $F=\partial H=\partial H^{\prime}$ inserted between them. There is a map

$$
\langle,\rangle: S(H) \times S\left(H^{\prime}\right) \longrightarrow S\left(S^{3}\right)=\mathbf{C},
$$

induced by the Kauffman bracket and the union of links. In [5] it was shown that, if $A$ is a primitive $4 r$-th root of unity, then

$$
W(F)=S(H) / \operatorname{ker}\langle,\rangle
$$

is the space associated to the surface $F$ by the $S U(2)$-TQFT at level $r$. Here ker denotes the left kernel of the bilinear form $\langle$,$\rangle . This space has however$ a more concrete description. If $i, j, k$ satisfy the following conditions:

$$
0 \leq i, j, k \leq r-1,|i-j| \leq k \leq i+j, i+j+k \text { is even, }
$$

then we can define an element of the skein space of the 3-ball with $i+j+$ $k$ boundary points, given by inserting $f^{(i)}, f^{(j)}, f^{(k)}$ in the diagram, and therefore connecting up with no crossings (see Figure 3 ).
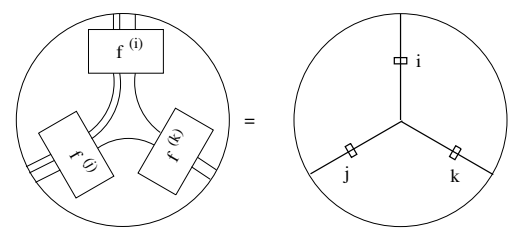

Figure 3. Vertex elements in the skein modules.

Now the triple $(i, j, k)$ is called admissible if, additionally to the previous conditions, it satisfies $0 \leq i, j, k \leq r-2$ and $i+j+k \leq 2(r-2)$. Furthermore let consider the standard 3 -valent graph in $H$ which is the standard spine of the handlebody $H$, and label its edges with integers $i_{1}, i_{2}, \ldots, i_{3 g-3}$, such that all labels incident to a vertex form an admissible triple. We form an element of $S(H)$ by inserting idempotents $f^{(i)}$ along the edges of the graph and triodes, like we did above at vertices. It is shown in $[\mathbf{5}, \mathbf{4 0}]$ that the vectors we obtain this way form a basis of the quotient space $W(F)$.

For a 3 -valent graph $\Gamma$, possibly with leaves and some of the edges already carrying a label, we denote by $W(\Gamma)$ the space generated by the set of labelings of (non-labeled) edges which have the property that all triples 
from incident edges are admissible. An easy extension of the arguments in $[\mathbf{5}, \mathbf{4 0}]$ shows that $W(\Gamma)$ is isomorphic to $W(F)$, provided that $\Gamma$ is some closed 3 -valent graph of genus $g$.

If $K$ and $K^{\prime}$ are the subgroups of the mapping class group $\mathcal{M}(F)$ of $F$ consisting of the classes of those homeomorphisms which extend to the handlebodies $H$ and $H^{\prime}$ respectively, then we have natural actions of $K$ on $S(H)$, and $K^{\prime}$ on $S\left(H^{\prime}\right)$. Moreover these actions descend to actions on the quotient $W(F)$. One of these two actions, say that of $K$ on $H$, has a simple meaning: Consider an element $x \in S(H)$, which is a representative of the class $[x] \in W(F), u \in K$, then $u(x)=[\varphi(x)] \in W(F)$, where $\varphi$ is a homeomorphism of $H$ whose restriction at $F$, modulo isotopy, is $u$. The other action, that of $K^{\prime}$ on $W(F)$ can be described in a similar manner, using the non-degenerate bilinear form $\langle$,$\rangle on W(F)$. Namely, $u(x)$, for $u \in K^{\prime}$, $x \in W(F)$ is defined by the equality:

$$
\langle u x, y\rangle=\left\langle x,\left[u\left(y^{\prime}\right)\right]\right\rangle
$$

holding for any $y \in W(F)$; on the right hand side $y^{\prime} \in S\left(H^{\prime}\right)$ is a lift of $y$, and the action of $K^{\prime}$ on $S\left(H^{\prime}\right)$ is the geometric one.

Moreover we have an induced action of the free group generated by $K$ and $K^{\prime}$ on $W(F)$. It is shown in $[40,34]$ that this action descends to a central extension of the mapping class group $\mathcal{M}(F)$. This is the representation coming from TQFT. Actually we can build up the TQFT starting from that representation. The main idea is that, if we cut a closed 3-manifold $M$ along a (closed embedded) surface $F$ into two pieces $M_{1}$ and $M_{2}$, then the invariant $Z(M)$ can be recovered from the invariants $Z\left(M_{i}\right)$ associated to $M_{i}$ (which are vectors in the space $W(F)$ ) as follows:

$$
Z(M)=\left\langle Z\left(M_{1}\right), Z\left(M_{2}\right)\right\rangle .
$$

If we want to glue back now $M_{1}$ to $M_{2}$ using an additional twist $\varphi \in \mathcal{M}(F)$ then we can compute also the invariant of the resulting manifold $M_{1} \cup_{\varphi} M_{2}$ using the (projective) representation $\rho: \mathcal{M}(F) \longrightarrow G L(W(F))$, defined above:

$$
Z\left(M_{1} \cup_{\varphi} M_{2}\right)=\left\langle\rho(\varphi) Z\left(M_{1}\right), Z\left(M_{2}\right)\right\rangle .
$$

We skipped the complications arising from the projective ambiguity, which is a root of unity, which amount to consider a supplementary structure (framing) on the manifold. This gives a simple formula for the invariant in terms of Heegaard splittings. In fact the vector $Z(H)=Z\left(H^{\prime}\right) \in W(F)$, associated to the handlebody is corresponding to the graph of genus $g$ whose labels are all 0 (up to a normalization factor, which we skip for simplicity). Then $Z\left(H \cup_{\varphi} H^{\prime}\right)$, the invariant of the closed manifold obtained by gluing two handlebodies along their common surface $F$ using the homeomorphism $\varphi$, is now $\left\langle\rho(\varphi) Z(H), Z\left(H^{\prime}\right)\right\rangle$. 
2.3. Transformation rules for planar diagrams in the skein modules.

In order to make explicit computations we will freely use the recipes from [35] which allows us to transform planar diagrams representing elements in the skein module of the 3-ball (with some boundary points) into simpler planar diagrams, eventually arriving to linear combinations of the elements of a fixed basis. For completeness we include these rules below.
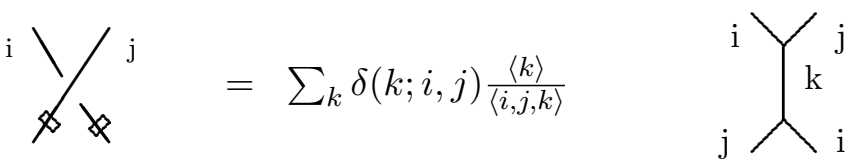

$i \bigcirc_{k}^{j} j$

$=\delta_{n}^{k} \frac{\langle i, j, k\rangle}{\langle k\rangle}$

$\mathrm{q}^{\mathrm{k}}$

i $\left.\right|^{j}$

$=\sum_{k} \frac{\langle k\rangle}{\langle i, j, k\rangle}$

$\bigcup_{j}^{i}{ }_{i}^{j}$

$\bigodot_{i}$

$=0$

for $k \geq 1$

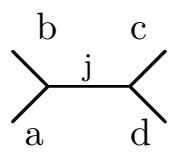

$=\sum_{i}\left\{\begin{array}{lll}a & b & i \\ c & d & j\end{array}\right\}$
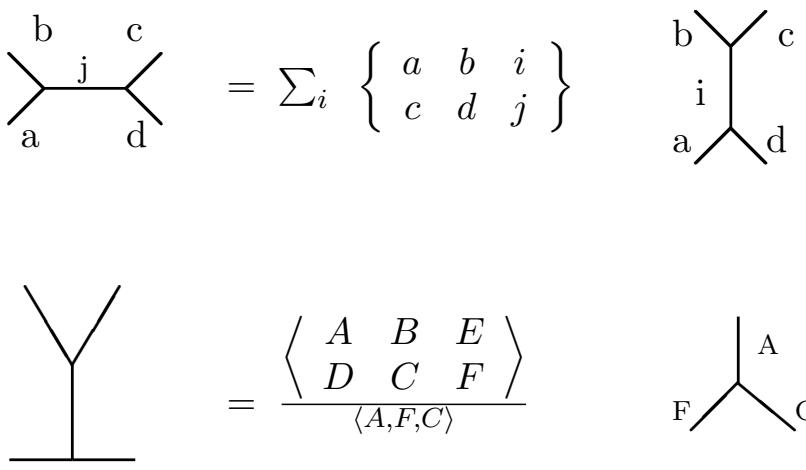

$=\frac{\left\langle\begin{array}{ccc}A & B & E \\ D & C & F\end{array}\right\rangle}{\langle A, F, C\rangle}$<smiles>CC(C)F</smiles> 

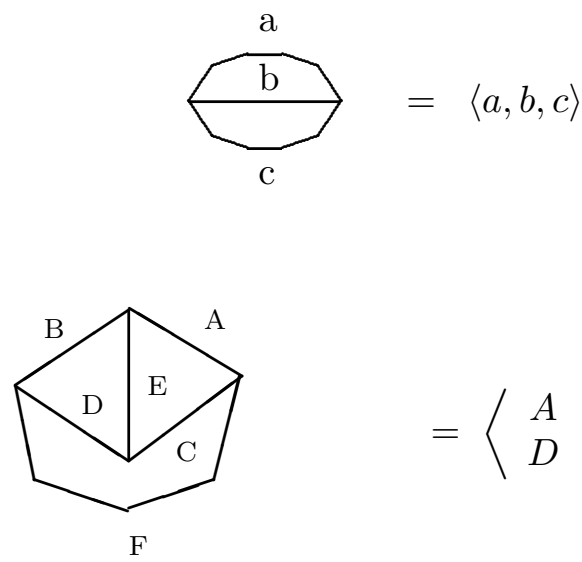

$$
=\left\langle\begin{array}{lll}
A & B & E \\
D & C & F
\end{array}\right\rangle
$$

where

$$
\begin{gathered}
\langle k\rangle=(-1)^{k}[k+1]=(-1)^{k} \frac{A^{2 k+2}-A^{-2 k-2}}{A^{2}-A^{-2}}, \\
\delta(c ; a, b)=(-1)^{k} A^{i j-k(i+j+k+2)}, \\
\langle a, b, c\rangle=(-1)^{i+j+k} \frac{[i+j+k+1] ![i] ![j] ![k] !}{[i+j] ![i+k] ![j+k] !} .
\end{gathered}
$$

Here $i, j, k$ are the internal colors given by

$$
i=\frac{b+c-a}{2}, j=\frac{a+c-b}{2}, k=\frac{b+a-c}{2},
$$

and $[n] !=[1][2] \ldots[n]$.

Consider now $A, B, C, D, E, F$ such that $(A, B, E),(B, D, F),(E, D, C)$, $(A, C, F)$ are admissible triples and make some notations: $\Sigma=A+B+$ $C+D+E+F, a_{1}=\frac{A+B+E}{2}, a_{2}=\frac{B+D+F}{2}, a_{3}=\frac{E+D+C}{2}, a_{4}=\frac{A+C+F}{2}$, $b_{1}=\frac{\Sigma-A-F}{2}, b_{2}=\frac{\Sigma-B-C}{2}, a_{1}=\frac{\Sigma-A-D}{2}$.

The tetrahedron coefficient is defined as:

$\left\langle\begin{array}{ccc}A & B & E \\ D & C & F\end{array}\right\rangle=\frac{\prod_{i} \prod_{j}\left[b_{i}-a_{j}\right] !}{[A] ![B] ![C] ![D] ![E] ![F] !}\left(\begin{array}{ccccccc}a_{1} & & a_{2} & & a_{3} & & a_{4} \\ & b_{1} & & b_{2} & & b_{3} & \end{array}\right)$,

where

$$
\left(\begin{array}{ccccccc}
a_{1} & & a_{2} & & a_{3} & & a_{4} \\
& b_{1} & & b_{2} & & b_{3} &
\end{array}\right)=\sum_{\max a_{j} \leq \zeta \leq \min a_{j}} \frac{(-1)^{\zeta}[\zeta+1] !}{\prod_{i}\left[b_{i}-\zeta\right] ! \prod_{j}\left[\zeta-a_{i}\right] !} .
$$

The quantum $6 \mathrm{j}$-symbol of [35] is given by the formula:

$$
\left\{\begin{array}{lll}
a & b & i \\
c & d & j
\end{array}\right\}=\frac{\langle i\rangle\left\langle\begin{array}{ccc}
i & b & c \\
j & d & a
\end{array}\right\rangle}{\langle i, a, d\rangle\langle i, b, c\rangle} .
$$




\section{Proof of the theorem.}

\subsection{Outline.}

Consider a surface $F$ of genus $g$ and let $\Gamma \subset H$ be a 3 -valent graph embedded in the handlebody $H$. Suppose that the graph $\Gamma^{\prime}$ shown in Figure 4 is a subgraph of $\Gamma$. Then $\Gamma^{\prime}$ can be viewed as the spine of the $(n+2)$-holed sphere $F^{\prime} \subset F$, which is the intersection of a regular neighborhood of $\Gamma^{\prime}$ (in $\left.\mathbf{R}^{3}\right)$ with $F$. Consider a partial labeling $\Gamma^{\prime}(n, m)$ of $\Gamma^{\prime}$ as shown on the right hand side in the figure. Notice that the leaf with label 0 can be removed without affecting the space $W\left(\Gamma^{\prime}(n, m)\right)$.

Lemma 3.1. For a suitably chosen $\Gamma$, of genus $g \geq 4$, there exist $m \geq$ $0, n \geq 5$, such that $W\left(\Gamma^{\prime}(n, m)\right) \subset W(\Gamma)$ and $\operatorname{dim} W\left(\Gamma^{\prime}(n, m)\right) \geq 2$. For $g=3$ we have $W\left(\Gamma^{\prime}(4,2)\right) \subset W(\Gamma)$, and for $g=2 W\left(\Gamma^{\prime}(3,1)\right) \subset W(\Gamma)$.

Proof. We connect among them the leaves of $\Gamma^{\prime}$ using some planar arcs, in order to obtain a closed graph of minimal genus, and such that the labels agree when making connections.

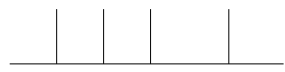

$\Gamma^{\prime}$

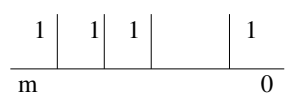

$\Gamma^{\prime}(\mathrm{n}, \mathrm{m})$

Figure 4. The graphs $\Gamma^{\prime}(n, m)$.

Fix now once for all the embedding of graphs $\Gamma^{\prime} \subset \Gamma$ as in the lemma, and denote by $V=V(n, m) \subset W(\Gamma)$ the image of $W\left(\Gamma^{\prime}(n, m)\right)$. Consider the curves $\gamma_{i j} \subset F^{\prime}, 1 \leq i, j \leq n$, drawn on the $(n+2)$-holed sphere $F^{\prime}$ which encircle the holes $i$ and $j$ like in Figure 5 and the set of curves $\gamma_{i}$ which are loops around the holes. The Dehn twists $T_{\gamma_{i j}}$ and $T_{\gamma_{i}}$ generate a subgroup $S$ of the mapping class group $\mathcal{M}(F)$.
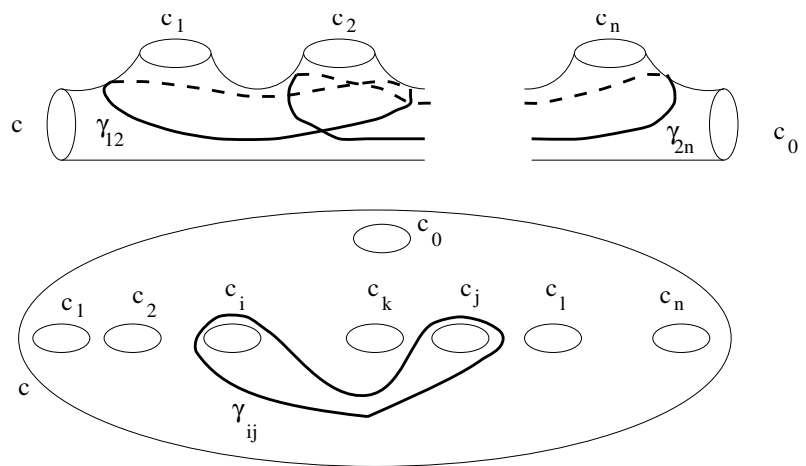

Figure 5. The curves $\gamma_{i j}$. 
Proposition 3.1. The subspace $V(n, m) \subset W(F)$ is $\rho(S)$-invariant. Moreover the image $\left.\rho\right|_{V(3,1)}(S) \subset G L(V(3,1))$ is an infinite group, provided that $g \geq 2$ and the level $r \neq 2,3,4,6,10$. For $r=10$ and $g \geq 3$ the image of $\left.\rho\right|_{V(4,2)}(S) \subset G L(V(4,2))$ is infinite.

The first part of the proposition follows from a more general fact concerning sub-surfaces $F^{\prime} \subset F$ and subgroups $S$ of $\mathcal{M}(F)$ of classes of homeomorphisms which keep $F^{\prime}$ invariant up to an isotopy, and send each boundary component into itself. Assume that a labeling of the boundary components of $F^{\prime}$ was fixed: This amounts to fix a labeling for the leaves of the subgraph $\Gamma^{\prime}$, the spine of $F^{\prime}$. Then the subspace $W\left(\Gamma^{\prime}\right) \subset W(\Gamma)$ is invariant by the action of $S$ on $W(F)$. Moreover, consider now that $F^{\prime}$ may be sent by a larger group $S^{\prime}$ into a sub-surface $F^{\prime \prime}$ which is isotopic to $F$, but the boundary components may be permuted among themselves. We claim now, that a subspace $W\left(\Gamma^{\prime}\right)$, associated to a labeling of the boundary components, is sent by such a homeomorphism into the subspace $W\left(\Gamma^{\prime}\right)$ associated to the permuted labeling on the boundary components. In particular the space $V$ from the proposition is not only invariant under $\rho(S)$, but also under larger groups which could permute the $n$ boundary components $c_{i}, i=1,2, \ldots, n$, since all their labels are identical.

Another observation is that the action of $\mathcal{M}\left(F^{\prime}\right)$ on the space $W\left(\Gamma^{\prime}\right)$, where $\Gamma^{\prime}$ has one external edge $e$ (corresponding to the boundary component $\left.c_{e} \subset \partial F^{\prime}\right)$ is the same as the action of $\mathcal{M}\left(F^{\prime} \cup_{c_{e}} D^{2}\right)$ on the space $W\left(\Gamma^{\prime \prime}\right)$; here $F^{\prime} \cup_{c_{e}} D^{2}$ is the result of gluing a disk on the circle $c_{e}$, and $\Gamma^{\prime \prime}$ is $\Gamma$ with the edge $e$ removed from it. This way we see that $\rho(S)$ acts like $\mathcal{M}\left(F^{\prime} \cup_{c_{0}} D^{2}\right)$ on the given space. This will help to find out the corresponding extension to the braid group.

Before we explain this action, remark that all Dehn twists along $\gamma_{i j}, \gamma_{i}$ are elements of the subgroup $K \subset \mathcal{M}(F)$ of classes of homeomorphisms extending to the handlebody $H$. Therefore, according to the discussion in the previous section, the action of $T_{\gamma_{i j}}$ (or $T_{\gamma_{i}}$ ) on $V(n, m)$ has a simple expression in the skein module of the 3-ball with $(n+2)$-boundary points: Just perform the Dehn twist on the 3-ball which is a regular neighborhood of the graph $\Gamma^{\prime}$, viewed as part of the handlebody $H$, whose spine is $\Gamma$. This is equivalent with twisting the $i$-th and $j$-th legs of the graph $\Gamma^{\prime}$. We have to apply further the skein relations, in order to compute the latter element in terms of the given basis of $V$, where all the legs are straight. Notice that the representative graphs considered in Sections 2.2 and 2.3 are framed graphs, and the framings considered in the planar pictures are the blackboard ones. When the Dehn twist $T_{\gamma_{i j}}$ acts on the spine graph $\Gamma^{\prime}$ then the framings of the strands $i$ and $j$ are altered. Then the action of $T_{\gamma_{i}}$ on the $i$-th strand is the change of its framing by one unit. Then the element $A_{i j}=T_{\gamma_{i j}} T_{\gamma_{i}}^{-1} T_{\gamma_{j}}^{-1}$ acts on $V(n, m)$ as follows: 


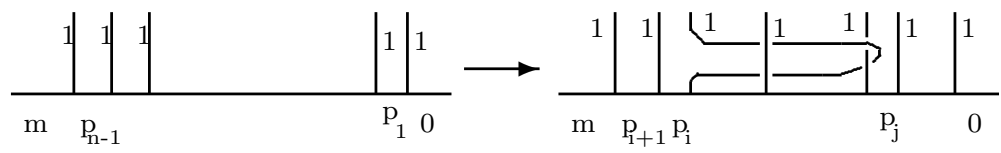

Remark that the $T_{\gamma_{i}}$ 's commute with all the other $T_{\gamma_{j k}}$ because their support curves can be made disjoint. These formulas make up a representation of the pure braid group $P_{n}$, which extends to the whole braid group in the obvious manner: Consider that the $i$-th and $i+1$-th legs are only half-twisted. This defines the action of the $i$-th generator $g_{i}$ of the braid group $B_{n}$. In fact, looking at the generators $A_{i j}$ of $P_{n}$ as elements of $B_{n}$, their action on $V$ consists in twisting the corresponding strands of $\Gamma^{\prime}$, modulo Reidemester moves in plane. On the other hand the fact that we obtained a representation of $B_{n}$ is checked the same manner: The relation $g_{i} g_{i+1} g_{i}=g_{i+1} g_{i} g_{i+1}$ translates into the third Reidemester move, which is obviously satisfied in the skein module. We continue to denote by $\rho$ the representation of $B_{n}$ on $V$. In fact this should enter in the computation of the action of elements in the larger group $\mathcal{M}(F)$, so actually is "part" of $\rho$ but for bigger genus.

The main ingredient in Proof of Proposition 3.1 is:

Proposition 3.2. The representations

$$
\left(-A^{-1}\right) \rho: B_{3} \longrightarrow \operatorname{End}(V(3,1))
$$

and respectively

$$
\left(-A^{-1}\right) \rho: B_{4} \longrightarrow \operatorname{End}(V(4,2))
$$

factor throughout the Temperley-Lieb algebras $A_{\beta, 3}$ (and respectively $A_{\beta, 4}$ ) where $\beta=2+q+q^{-1}$ and the parameter $q=A^{-4}$.

Let us explain what is meant by $c \rho$ where $c \in \mathbf{C}$ is a constant. This is another representation of the braid group, which is defined on the generators by $(c \rho)\left(g_{i}\right)=c \rho\left(g_{i}\right)$. Therefore if $w$ is a word in the generators $g_{i}$ we have $(c \rho)(w)=c^{|w|} \rho(w)$, where $|w|$ is the sum of exponents appearing in the word $w$. Since the braid relations are homogeneous in the generators $g_{i}$ this is well-defined.

We will prove that the image of the braid group is infinite in $A_{\beta, n}$. This was done by Jones in [20] for one value of $A$ (and a slightly different context), but the proof extends to an arbitrary primitive root of unity. We can be more precise. Let us consider $M_{2}(\mathbf{C})$ as the image of $A_{\beta, 3}$ by the natural projection, and the factor $M_{3}(\mathbf{C})$ as the quotient of $A_{\beta, 4}$, when both are semi-simple.

Proposition 3.3. The image of $B_{3}$ in $M_{2}(\mathbf{C})\left(\right.$ via $\left.A_{\beta, 3}\right)$ is infinite provided that $r \neq 2,3,4,6,10$. For $r=10$ the image of $B_{4}$ in $M_{3}(\mathbf{C})\left(\right.$ via $\left.A_{\beta, 4}\right)$ is infinite. 
Now the algebra $A_{\beta, n}(n=3,4)$ is semi-simple for $r$ outside the excepted range $r \neq 3,4$ and $A_{\beta, 3}=M_{2}(\mathbf{C}) \oplus \mathbf{C}$. The representation $\tilde{\rho}$ is not abelian and then the induced map $A_{\beta, 3} \longrightarrow M_{2}(\mathbf{C})=\operatorname{End}(V(3,1))$ must be the canonical projection (up to an automorphism). Thus the image of $B_{3}$ by $\rho$ should be infinite. For $r=10$ we work within $B_{4}$ and $A_{\beta, 4}$ and we are forced then to restrict to $g \geq 3$. Again $\tilde{\rho}$ is not abelian and we will see in the next section that it is irreducible. Therefore the induced map $A_{\beta, 4} \longrightarrow$ $M_{3}(\mathbf{C})=\operatorname{End}(V(4,2))$ is again the projection on the corresponding factor. This establishes Proposition 3.1, because $P_{n}$ is of finite index in $B_{n}$.

Eventually recall that $\rho$, at the mapping class group level, is only a projective representation, and it can be also considered as a representation of a finite extension (depending on the level) of $\mathcal{M}_{g}$. The image group stays then in the unitary group modulo roots of unity of order $4 r$ and thus the claim of Theorem 1.1 (concerning the BHMV-version) is proved. The present proof (see Section 3.3) shows actually that the image in the projective unitary group (i.e. modulo $U(1)$ ) is also infinite.

\subsection{Proof of Proposition 3.2.}

The TQFT considered here is the one constructed in [5], for $A$ a primitive $2 r$-th root of unity. We suppose for simplicity that $r$ is even, hence we are working with the $S U(2)$-TQFT. The same arguments hold verbatim for the representation associated to the $S O(3)$-TQFT, with only minor modifications in the range of colors.

Lemma 3.2.1. A basis for $V(n, m)$ is provided by the labeled graphs $L(\mathbf{p})$ below,

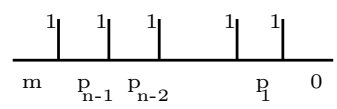

whose labels are in one-to-one correspondence with

$$
\begin{aligned}
B(V)=\left\{\mathbf{p}=\left(p_{0}, p_{1}, \ldots, p_{n}\right) ; p_{i} \in \mathbf{Z}_{+},\right. \\
\left.\quad p_{0}=0, p_{n}=m, p_{i} \leq 2 r-2,\left|p_{i}-p_{i+1}\right|=1, i=0, \ldots, n\right\} .
\end{aligned}
$$

Proof. It follows immediately from the admissibility conditions on the triples $\left(p_{i}, p_{i+1}, 1\right)$.

Then the computation of $\rho\left(g_{i}\right)$ is reduced to that of $g_{i} L(\mathbf{p})$, in the skein module. Observe now that the only values of the labels $p_{j}$ which may change when $g_{i}$ is applied are $p_{i-1}, p_{i}, p_{i+1}$. This will be also seen during the explicit computation.

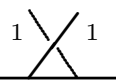

Actually we have to compute $\overline{\mathrm{a}}$ b c and according to the lemma it suffices to consider that $|a-b|=|b-c|=1$. 
Lemma 3.2.2. Suppose that $|a-b|=|b-c|=1$ and $|a-c|=2$. Then

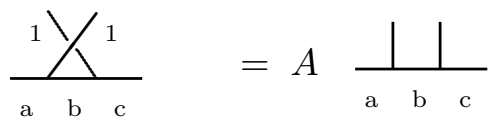

Proof. Suppose for simplicity that $c=a+2, b=a+1$. Then according to [35] and Section 2.3 we have

$$
\frac{1 \backslash\langle 1}{\mathrm{a} a+1 \mathrm{a}+2}=\sum_{k} \delta(k ; 1,1) \frac{\langle k\rangle}{\langle 1,1, k\rangle}
$$

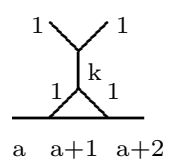

Therefore the triple $(1,1, k)$ has to be admissible, so that $k \in\{0,2\}$. Also the open tetrahedron

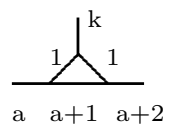

vanishes if $(k, a, a+2)$ is not admissible, so that there is only one possibility left, namely $k=2$. We get rid of the triangular face by using the formula:

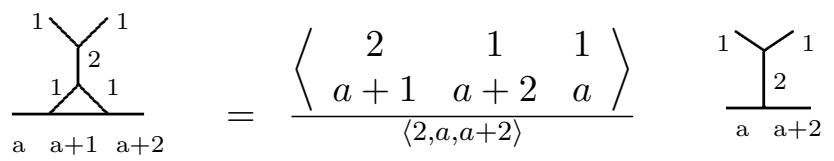

We eventually perform a fusion in order to express the right hand side in terms of the the usual basis $L(\mathbf{p})$. The formula of the fusing is:

$$
\frac{\left.{ }^{1}\right|^{1}}{\mathrm{a} a+1 \mathrm{a}+2}=\left\{\begin{array}{ccc}
a & 1 & 2 \\
1 & a+2 & a+1
\end{array}\right\} \quad \begin{aligned}
& { }^{1}{ }_{2}^{1} \\
& \mathrm{a} \mathrm{a}+2
\end{aligned}
$$

where the quantum 6-j symbol involved, namely $\left\{\begin{array}{ccc}a & 1 & 2 \\ 1 & a+2 & a+1\end{array}\right\}$, it turns out to be equal to 1 , for all $a$. This implies that

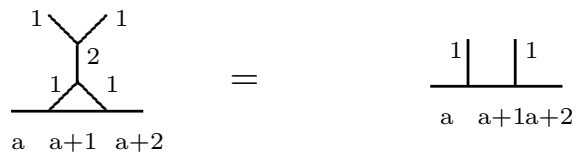

and the lemma follows.

Lemma 3.2.3. The following identities hold: 
$\frac{1 \backslash / 1}{\mathrm{a} \text { a-1 a }}-A \frac{A^{-4}[a]-[a+2]}{[2][a+1]}=\frac{1||^{1}}{\text { a } \mathrm{a}-1 \quad \mathrm{a}}+A \frac{\left(A^{-4}+1\right)[a+1]}{[a+2]+[a]} \underset{\mathrm{a} \quad \mathrm{a}+1 \mathrm{a}}{1}$

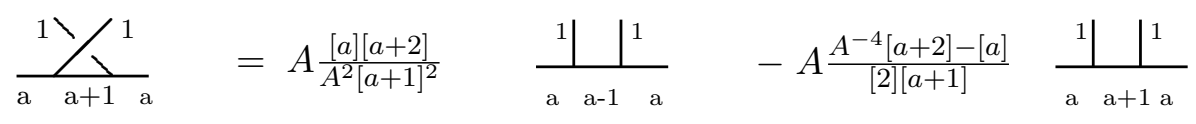

Here is to be understood that for $a=2 r-2$ we have $\frac{1 \mid{ }^{1}}{\mathrm{a} a+1 \mathrm{a}} \quad 0, \quad$ and for $a=0$ the equality $\frac{1||_{\text {a } \mathrm{a}-1 \text { a }}^{1}}{0,}, \quad$ holds.

Proof. We have, like in the previous lemma, the following formula:

$$
\begin{aligned}
& \frac{1 \backslash / \backslash 1}{\mathrm{a} b \mathrm{~b}}=\frac{A^{-3}}{[2]} \\
& \frac{A^{-3}}{[2]} \frac{\left\langle\begin{array}{ccc}
0 & 1 & 1 \\
b & a & a
\end{array}\right\rangle}{\langle 0, a, a\rangle}
\end{aligned}
$$
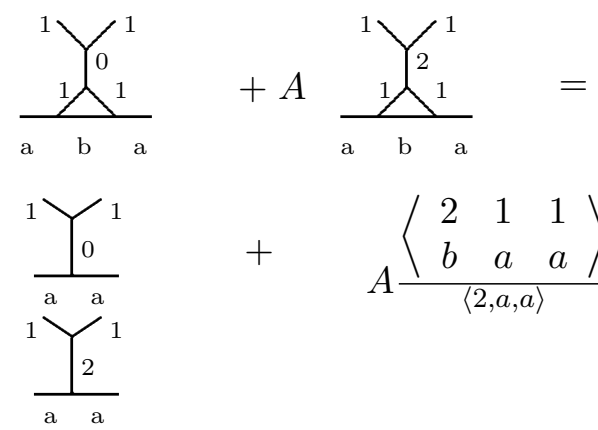

We have to perform a fusing, in order to arrive to the standard basis of $V$. Using the computations of $6 \mathrm{j}$-symbols appearing in this particular fusing we obtain that:

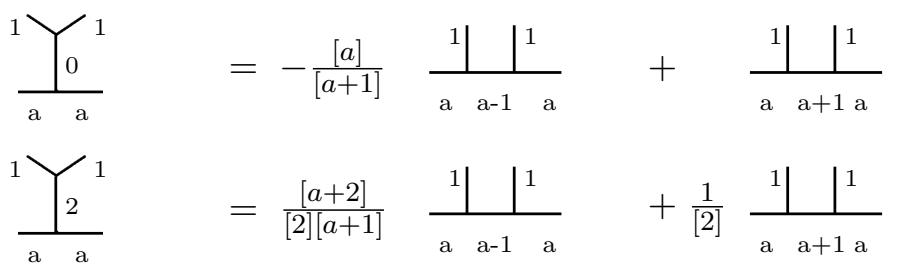

The convention is that diagrams whose labels form non-admissible triples are vanishing. Explicit computations now yield our claim.

Lemma 3.2.4. The following numerical identities are satisfied:

$$
[a]+[a+2]=[2][a+1], \frac{[a+1]\left(1+A^{-4}\right)}{[a]+[a+2]}=\frac{1}{A^{2}},
$$




$$
\frac{[a+2] A^{-4}-[a]}{[2][a+1]}=\frac{A^{-4}-1}{1-A^{4+4 a}}, \frac{[a] A^{-4}-[a+2]}{[2][a+1]}=\frac{A^{-4}-1}{1-A^{-4-4 a}} .
$$

The proof is a mere computation.

Set now $q=A^{-4}$. Then Lemma 3.2.3 can be reformulated as

$\rho\left(g_{i}\right) \frac{1||^{1}}{\text { a a-1 a }}=-A \frac{q-1}{1-q^{a+1}} \quad \frac{1||^{1}}{\text { a a-1 a }}+\frac{1}{A}$

$\rho\left(g_{i}\right) \frac{\left.{ }^{1}\right|^{1}}{\text { a a } \mathrm{a}+1 \mathrm{a}} \quad=\nu^{2} A^{3} \quad \frac{1||^{1}}{\mathrm{a} \text { a-1 a }} \quad-A \frac{q-1}{1-q^{-a-1}}$
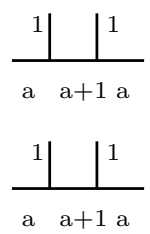

where $\nu^{2}=\frac{\left(1-q^{a+2}\right)\left(1-q^{a+1}\right)\left(1-q^{-a}\right)\left(1-q^{-a-1}\right)}{(1+q)^{2}}$.

We specify now to the cases $n=3, m=1$, and $n=4, m=2$. Then the vector space $V(n, m)$ has dimension 2 and respectively 3 . The Hecke algebra relations follow immediately from the formulas above. The additional relation defining $A_{\beta, n}$ is verified by a direct computation.

Let us check out the case $n=3$. The two vectors which span $V(3,1)$ have the labels $\left(0, p_{1}, p_{2}, m\right) \in\{(0,1,0,1),(0,1,2,1)\}$. Then the previous formulas read:

$$
\begin{gathered}
\tilde{\rho}\left(g_{1}\right)=\left(\begin{array}{cc}
-1 & 0 \\
0 & A^{-4}
\end{array}\right), \\
\tilde{\rho}\left(g_{2}\right)=\left(\begin{array}{cc}
\frac{1}{A^{4}\left(1+A^{4}\right)} & -\frac{A^{4}+A^{-4}+1}{A^{2}\left(A^{4}+A^{-4}+2\right)} \\
-A^{-2} & -\frac{1}{1+A^{-4}}
\end{array}\right),
\end{gathered}
$$

where $\tilde{\rho}=\left(-A^{-1}\right) \rho$. This implies that

$$
\begin{aligned}
& \tilde{\rho}\left(g_{1} g_{2}\right)=\left(\begin{array}{cc}
-\frac{1}{A^{4}\left(1+A^{4}\right)} & \frac{A^{4}+A^{-4}+1}{A^{2}\left(A^{4}+A^{-4}+2\right)} \\
-A^{-6} & -\frac{1}{A^{4}\left(1+A^{-4}\right)}
\end{array}\right), \\
& \tilde{\rho}\left(g_{2} g_{1}\right)=\left(\begin{array}{cc}
-\frac{1}{A^{4}\left(1+A^{4}\right)} & -\frac{A^{4}+A^{-4}+1}{A^{6}\left(A^{4}+A^{-4}+2\right)} \\
A^{-2} & -\frac{1}{A^{4}\left(1+A^{-4}\right)}
\end{array}\right),
\end{aligned}
$$

and

$$
\tilde{\rho}\left(g_{1} g_{2} g_{1}\right)=\left(\begin{array}{cc}
\frac{1}{A^{4}\left(1+A^{4}\right)} & \frac{A^{4}+A^{-4}+1}{A^{6}\left(A^{4}+A^{-4}+2\right)} \\
A^{-6} & -\frac{1}{A^{8}\left(1+A^{-4}\right)}
\end{array}\right) .
$$

Therefore the relation $1+\tilde{\rho}\left(g_{1}\right)+\tilde{\rho}\left(g_{2}\right)+\tilde{\rho}\left(g_{1} g_{2}\right)+\tilde{\rho}\left(g_{2} g_{1}\right)+\tilde{\rho}\left(g_{1} g_{2} g_{1}\right)=0$ holds, which proves the first part of Proposition 3.1.

Let us give the explicit matrices for $n=4, m=2$ and an ad-hoc proof of the irreducibility. The space $V(4,2)$ is spanned by the vectors of labels 
$\left(0, p_{1}, p_{2}, p_{3}, m\right) \in\{(0,1,0,1,2),(0,1,2,1,2),(0,1,2,3,2)\}$. Then within this basis we have from above:

$$
\begin{gathered}
\tilde{\rho}\left(g_{1}\right)=\left(\begin{array}{ccc}
A^{-4} & 0 & 0 \\
0 & -1 & 0 \\
0 & 0 & -1
\end{array}\right), \\
\tilde{\rho}\left(g_{2}\right)=\left(\begin{array}{ccc}
-\frac{A}{1+A^{-4}} & -A^{-2} & 0 \\
-\frac{\left(1-A^{12}\right)\left(1-A^{4}\right)^{3}}{A^{34}} & \frac{1}{1+A^{8}} & 0 \\
0 & 0 & -1
\end{array}\right), \\
\tilde{\rho}\left(g_{3}\right)=\left(\begin{array}{ccc}
-1 & 0 & 0 \\
0 & -\frac{1}{1+A^{-4}+A^{-8}} & -A^{-2} \\
0 & -\frac{\left(1+A^{8}\right)^{2}\left(A^{4}-1\right)^{2}\left(A^{12}-1\right)^{2}}{A^{66}} & \frac{A^{-12}}{1+A^{-4}+A^{-8}}
\end{array}\right) .
\end{gathered}
$$

Assume that the $B_{4}$ representation $\tilde{\rho}$ is not irreducible. Then the $A_{\beta, 4^{-}}$ module $V(4,2)$ is completely reducible and there is at least one simple factor of dimension 1. Equivalently, $V(4,2)$ contains a 1-dimensional $B_{4}$-invariant subspace say $\mathbf{C} w$, for some non-zero vector $w$. There exist then the scalars $\lambda_{i}$ such that $\tilde{\rho}\left(g_{i}\right) w=\lambda_{i} w$. The group relations imply $\lambda_{1}=\lambda_{2}=\lambda_{3}=\lambda$ (since the matrices above are non-singular) and from the relations in $A_{\beta, 4}$ we derive $\lambda=-1$. However the condition $\tilde{\rho}\left(g_{1}\right) w+w=0$ yields $w_{1}=0$, further $\tilde{\rho}\left(g_{2}\right) w+w=0$ adds the constraint $w_{2}=0$ and the last identity shows that $w$ vanishes. This proves that $V(4,2)$ is irreducible. In particular $\tilde{\rho}$ factors throughout the projection $A_{\beta, 4} \longrightarrow M_{3}(\mathbf{C})$.

Remarks 3.2.5. We could show from the very beginning of the proof that the representation of $B_{n}$ factors through the Hecke algebra $H_{n}(q)$ with $q=$ $A^{-4}$. Let assume we are interested in the action of $g_{j}$. Observe that the vectors
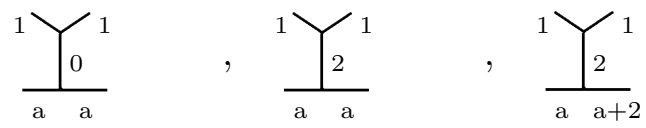

and the corresponding ones with $a+2$ replaced by $a-2$, (having the vertical strand on the $j$-th position) span all of $V(n, m)$. Indeed using the fusing matrices (which are invertible) we can relate this system to the standard basis $L(\mathbf{p})$.

But now these are precisely the eigenvectors for $g_{i}$, because we have the following relations:

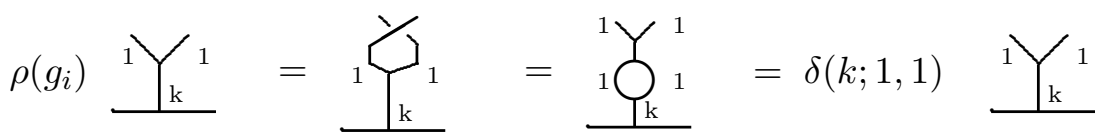


This implies that the eigenvalues of $\rho\left(g_{i}\right)$ are $-A^{-3}$ and $A$, so that shifting $\rho$ by a factor of $-A^{-1}$ will change them into -1 and $A^{-4}$, as in the usual presentation of $H_{n}(q)$ with $q=A^{-4}$.

It can be proved (but this is beyond the scope of this note) that the representation $\tilde{\rho}$ is precisely the Hecke algebra representation $\pi_{\lambda}^{(r)}$ associated to the Young diagram $\lambda=\left[\frac{n+m}{2}, \frac{n-m}{2}\right]$ as considered by Wenzl in [49]. This is clear for $n=3, m=1$. The direct computational approach is somewhat cumbersome for the general case. However from [3] we can derive a still more general equivalence between the Hecke algebras representation associated to a Young diagram $\lambda$ with $N$ rows and that arising in the previous construction for the $S U(N)$-TQFT, where the label $m$ is replaced by the "color" $\lambda$. This follows from the irreducibility of the latter, using the technique from [3].

\subsection{Proof of Proposition 3.3.}

In [20] a proof for Proposition 3.3 is given for the case when $q=$ $\exp \left(\frac{2 \pi \sqrt{-1}}{r}\right)$, but the argument generalizes easily to all primitive roots of unity. We outline it below, for the sake of completeness.

It is known that $A_{\beta, 3}$ is semi-simple and splits as $A_{\beta, 3}=M_{2}(\mathbf{C}) \oplus \mathbf{C}$, for all $\beta \neq 1$ (see the Theorem 2.8.5, p. 98 from [16]). It suffices then to show that the images $\pi\left(g_{1}\right)$ and $\pi\left(g_{2}\right)$ in the factor $M_{2}(\mathbf{C})$ generate an infinite group. Observe first that $\pi\left(g_{1}\right)$ and $\pi\left(g_{2}\right)$ (and respectively $\tilde{\rho}\left(g_{1}\right)$ and $\tilde{\rho}\left(g_{2}\right)$ ) do not commute with each other. Thus the $A_{\beta, 3}$-module $V(3,1)$ is isomorphic to the simple non-trivial factor $M_{2}(\mathbf{C})$. As a consequence it suffices to see what happens with the images of these two generators, when restricted to this summand. The $B_{3}$ representation on $M_{2}(\mathbf{C})$ is also unitarizable when $q$ is a root of unity according to Proposition 3.2, p. 257 from [20]. Thus it makes sense to consider the images $\iota\left(g_{1}\right)$ and $\iota\left(g_{2}\right)$ in $S O(3)=U(2) / \mathbf{C}^{*}$. We have then the following decomposition in orthogonal projectors:

$$
\iota\left(g_{i}\right)=q e_{i}-\left(1-e_{i}\right),
$$

so that the order of $\iota\left(g_{i}\right)$ in $S O(3)$ is $2 r$ if $r$ is odd, $r / 2$ if $r=2(4)$ and $r$ if $r=0(4)$, because $q$ is a primitive root of unity of order $r$. As $r \neq 1$ these two elements cannot belong to a cyclic or dihedral subgroup of $S O(3)$. But no other subgroups have elements of order bigger than 5 . Thus for $r=5,7,8,9$ or $r \geq 11$, the image in $M_{2}(\mathbf{C})$ of the subgroup generated by $g_{1}$ and $g_{2}$ is infinite.

When $r=10$ we have to work within $B_{4}$, likewise to [20], p. 269. We already saw that the $A_{\beta, 4}$-module $V(4,2)$ is irreducible and identified therefore with the simple factor $M_{3}(\mathbf{C})$ from $A_{\beta, 4}$. Moreover the representation of $B_{4}$ on this factor was explicitly found out in [19], and it is the tensor product of the Burau and parity representations. It is also shown that the Burau representation contains elements of infinite order, for instance $g_{1} g_{2} g_{3}^{-1}$. 
We may wonder whether an element of infinite order in the image can be explicitly given for $r \neq 10$. Since we have to consider only the matrices $\iota\left(B_{n}\right)$ in $S O(3)$, it is very likely that the element $g_{1}^{-1} g_{2}$ has infinite order.

Remark 3.1. Once we obtained the fact that the image of $\mathcal{M}_{g}$ is infinite at a particular primitive root of unity, we may argue also as follows: The Galois group $\operatorname{Gal}(\overline{\mathbf{Q}} ; \mathbf{Q})$ acts on the set of roots of unity, as well as on the entries of the matrices $\rho(x)$, with $x \in \mathcal{M}_{g}$. It suffices to prove that the two actions of $\operatorname{Gal}(\overline{\mathbf{Q}} ; \mathbf{Q})$ are compatible to each other, in order to conclude that the image group is infinite at all roots of unity. This argument was pointed to me by Gregor Masbaum.

\subsection{The RT version.}

Lickorish [31] established the relationship among the invariants obtained via the Temperley-Lieb algebra (basically those from [4]) $I(M, A)$ and the Reshetikhin-Turaev invariant $\tau_{r}(M)$ (see [25]), for closed oriented 3-manifolds $M$, as follows:

$$
I\left(M,-\exp \frac{\pi \sqrt{-1}}{2 r}\right)=\exp \left(\frac{(6-3 r) b_{1}(M) \pi \sqrt{-1}}{4 r}\right) \tau_{r}(M),
$$

where $b_{1}(M)$ is the first Betti number of the manifold $M$. Roughly speaking the two invariants are the same up to a normalization factor. There are however two associated TQFTs, still very close to each other:

1) The TQFT based on the Kauffman bracket, as described in [5], which arises in a somewhat canonical way; in fact any invariant of closed 3 -manifolds extends to a TQFT via this procedure (see $[\mathbf{5}, \mathbf{1 0}]$ for details). The associated mapping class group representation we denote it by $\rho^{K}$.

2) The TQFT based on the Jones polynomial, as described in [25] (see also [13]). The associated mapping class group representation we denote it by $\rho^{J}$, and may be computed using the definitions from conformal field theory like in [37]. A derivation of this representation, and the reconstruction of the invariant from it was first given by Kohno $[27]$ (see also $[44,45,12]$ ).

The two representations are similar: The associated spaces on which they act are naturally isomorphic. This means that in both theories $W(F)$ has a distinguished basis given by labelings of 3 -valent graphs, with the same set of labels. Basically both theories are built up using some variants of the quantum $6 \mathrm{j}$-symbols:

1) In [35] these are identified with the tetrahedron coefficients, (see also $[\mathbf{2 3}]$ ); the relationship with the usual $6 \mathrm{j}$-symbol (coming from representation theory) was outlined in [38].

2) In the case of $\rho^{J}$ the $6 \mathrm{j}$-symbols are coming from the representation theory of $U_{q}\left(s l_{2}\right)$ and where described in $[\mathbf{2 6}]$. 
Consider now the analogous subspace $V(n, m)=W\left(\Gamma^{\prime}(n, m)\right)$ of $W(F)$, as in 3.1. We have again an action of the braid group $B_{n}$ on $V$, but this time the interpretation is no longer related to skein modules of the ball. Here the graph $\Gamma$ is considered to be embedded in the surface $F$, giving a rigid structure on $F[\mathbf{1 0}, \mathbf{4 8}]$. This means that there is a pants decomposition $c$ of $F$ with the property that all circles in $c$ are transversal to $\Gamma$, the intersection of $\Gamma$ with every trinion is the suspension of 3 points (topologically, the space underlying the figure $Y$ ). Remark that $c$ and $\Gamma$ determine uniquely an identification of $F$ with a fixed and decomposed surface, up to an isotopy.

This time twisting the strands of the labeled graphs in $L(\mathbf{p})$ can be expressed in terms of the data of conformal field theory (see [27]). Specifically, we have:

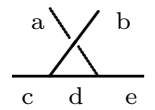

$$
=\sum_{j} B_{d j}\left[\begin{array}{ll}
a & b \\
c & e
\end{array}\right]
$$

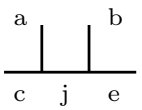

where the matrix $B$ is the so-called braiding matrix. The braiding matrix can be expressed in terms of the fusing matrix $F$ (see $[27,37]$ ) by the following formula:

$$
\begin{aligned}
& B_{i j}\left[\begin{array}{ll}
j_{2} & j_{3} \\
j_{1} & j_{4}
\end{array}\right] \\
& =(-1)^{j_{1}+j_{4}-i-j} / 2 \exp \left(\pi \sqrt{-1}\left(\Delta_{j_{1}}+\Delta_{j_{4}}-\Delta_{i}-\Delta_{j}\right)\right) F_{i j}\left[\begin{array}{ll}
j_{1} & j_{3} \\
j_{2} & j_{4}
\end{array}\right],
\end{aligned}
$$

where

$$
\Delta_{j}=\frac{j(j+1)}{4 r} .
$$

We use the same set of labels for the graphs, namely integers running from 1 to $2 r-2$ as before, instead of the traditional half-integer labels from $[\mathbf{2 6}, \mathbf{2 7}, \mathbf{2 3}]$. Set also $q=\exp \frac{2 \pi \sqrt{-1}}{r}$, and $[n]=\frac{q^{n}-q^{-n}}{q-q^{-1}}$.

The natural choice for the fusing matrix $F$ is (see $[\mathbf{2 7}]$, p. 213-214, [46]):

$$
F_{i j}\left[\begin{array}{ll}
j_{1} & j_{3} \\
j_{2} & j_{4}
\end{array}\right]=\left\{\begin{array}{lll}
j_{1} & j_{3} & i \\
j_{2} & j_{4} & j
\end{array}\right\}_{K R},
$$

where $\{,\}_{K R}$ denotes the quantum 6j-symbols of Kirillov and Reshetikhin.

Using the computations from [26], and those from 2.3 we find that the only non-trivial braiding matrix for $j_{2}=j_{3}=1$ is that with $j_{1}=j_{4}$, and its value is therefore:

$$
B\left[\begin{array}{ll}
1 & 1 \\
a & a
\end{array}\right]=\left(\begin{array}{cc}
-q^{a+\frac{1}{4}}\left(\frac{[a]}{[2][a+1]}\right)^{1 / 2} & -q^{-\frac{1}{4}}\left(\frac{[a+2]}{[2][a+1]}\right)^{1 / 2} \\
-q^{-\frac{1}{4}}\left(\frac{[a+2]}{[2][a+1]}\right)^{1 / 2} & -q^{-a-\frac{3}{4}}\left(\frac{[a]}{[2][a+1]}\right)^{1 / 2}
\end{array}\right) .
$$

Notice that the braiding matrices arising in conformal field theory were previously computed by Tsuchyia and Kanie in [42]. Their result, used 
however a different normalization and the matrices are not identical, but equivalent up to a power of $q$. In fact, in our case, the representation $q^{1 / 4} \rho^{J}$ is also equivalent to $\tilde{\rho}$, for $n=3,4$. As an immediate consequence the representation $\rho^{J}$ has an infinite image, too, under the same condition as $\rho^{K}$. This ends the proof of the main theorem.

\section{References}

[1] L.C. Biederharn and J.D. Louck, Angular momentum in Quantum Physics, Encycloped. Math. and Its Appl., no. 8, Addison-Wesley, 1981.

[2] J. Birman, Braids, links and mapping class groups, Ann. Math. Studies, Princeton Univ. Press, no. 82, 1974.

[3] C. Blanchet, Hecke algebras and 3-manifolds quantum invariants, Preprint, 1997.

[4] C. Blanchet, N. Habegger, G. Masbaum and P. Vogel, Three-manifold invariants derived from the Kauffman bracket, Topology, 31 (1992), 685-699.

[5] _ Topological quantum field theories derived from the Kauffman bracket, Topology, 34 (1995), 883-927.

[6] C. Blanchet and G. Masbaum, Topological quantum field theories for surfaces with spin structures, Duke Math. J., 82 (1996), 229-267.

[7] N. Bourbaki, Algèbres de Lie, IV, V, VI, Masson, 1981.

[8] W. Eholzer, Fusion algebras induced by representations of the modular group, Internat. J. Modern Phys. A, 8(20) (1993), 3495-3507.

[9] W. Eholzer and N.P.Skoruppa, Conformal characters and theta series, Lett. Math. Phys., 35 (1995), 197-211.

[10] L. Funar, 2+1-D Topological quantum field theory and 2-D conformal field fheory, Commun. Math. Phys., 171 (1995), 405-458.

[11] _ Thêta functions, root systems and 3-manifold invariants, J. Geom. Phys., 17 (1995), 61-82.

[12] _ TQFT for general Lie algebras and open 3-manifolds, J. Math. Sci. Univ. Tokyo, 4 (1997), 121-181.

[13] R. Gelca, The $S L(2, C)$ Topological Quantum Field Theory with corners, Preprint q-alg/9604006, 1996.

[14] P. Gilmer, On the WRT representations of the mapping class groups, Proc. A.M.S., to appear.

[15] T. Gocho, The topological invariant of three manifolds based on the $U(1)$ gauge theory, J. Fac. Sci. Univ. Tokyo, Sect. 1A, Math., 39 (1992), 169-184.

[16] F.M. Goodman, P. de la Harpe and V.F.R. Jones, Coxeter graphs and tower of algebras, MSRI Series, Berkeley, Springer, New-York, 1989.

[17] S.P. Humphries, Normal closures of powers of Dehn twists in mapping class groups, Glasgow J. Math., 34 (1992), 313-317.

[18] L. Jeffrey, Chern-Simons-Witten invariants of lens spaces and torus bundles, Commun. Math. Phys., 147 (1992), 563-604.

[19] V.F.R. Jones, Hecke algebras and a polynomial invariant for knots, Ann. of Math., 126 (1987), 335-388. 
$[20]$ Braid groups, Hecke algebras and type $\mathrm{II}_{1}$-factors, in 'Geometric methods in operator algebras', Proc. US-Japan Seminar, H. Araki, E.G. Effros Eds., 1986, 242-271.

[21] V. Kac, Infinite dimensional Lie algebras, Progress in Math., 1983.

[22] V.G. Kac and D.H. Petersen, Infinite dimensional Lie algebras, theta functions and modular forms, Adv. Math., 53 (1984), 125-264.

[23] L. Kauffman and S.L. Lins, Temperley-Lieb Recoupling Theory and Invariants of 3-manifolds, Ann. Math. Studies 134, Princeton Univ. Press, 1994.

[24] T. Kerler, Mapping class group actions on quantum doubles, Commun. Math. Phys., 168 (1995), 353-388.

[25] R. Kirby and P. Melvin, The 3-manifold invariants of Witten and Reshetikhin-Turaev for $\operatorname{sl}(2, C)$, Invent. Math., 105 (1991), 473-545.

[26] A.N. Kirillov and N.Yu. Reshetikhin, Representation of the algebra of $U_{q}\left(s l_{2}\right), q-$ orthogonal polynomials and invariants for links, in 'Infinite dimensional Lie algebras and groups', Adv. Ser. Math. Phys., V. Kac, Editor, 1988, 285-339.

[27] T. Kohno, Topological invariants for 3-manifolds using representations of mapping class groups I, Topology, 31 (1992), 203-230.

[28] _ Topological invariants for 3-manifolds using representations of mapping class groups II, Contemporary Math., 175 (1994), 193-217.

[29] B.H. Li and T.J. Li, Generalized Gaussian sums and Chern-Simons-Witten-Jones invariants of lens spaces, J. Knot Theory and Its Ramif., 5 (1996), 183-224.

[30] R. Lickorish, Three-manifold invariants and the Temperley-Lieb algebra, Math. Annalen., 290 (1991), 657-670.

[31] _ Calculations with the Temperley-Lieb algebra, Comment. Math. Helv., 67 (1992), 571-591.

[32] _ Sampling the SU(n)-invariants, J. Knot Theory and Its Ramif., 6 (1997), 45-60.

[33] D. Littlewood, The theory of group characters, Oxford Univ. Press, 1940.

[34] G. Masbaum and J. Roberts, On central extensions of mapping class groups, Math. Annalen., 302 (1995), 131-150.

[35] G. Masbaum and P. Vogel, 3-valent graphs and the Kauffman bracket, Pacific J. Math., 164 (1994), 361-381.

[36] S.D. Mathur and A. Sen, Group theoretic classification of rational conformal field theories with algebraic characters, Nucl. Phys. B, 327 (1989), 725-743.

[37] G. Moore and N. Seiberg, Classical and quantum field theory, Commun. Math. Phys., 123 (1989), 177-254.

[38] S. Piunikhin, Reshetikhin-Turaev and Kontsevich-Kohno-Crane invariants for 3manifolds coincide, J. Knot Theory and its Ramif., 2 (1993), 65-95.

[39] N. Reshetikhin and V. Turaev, Invariants of three-manifolds via link polynomials and quantum groups, Invent. Math., 103 (1991), 547-597.

[40] J. Roberts, Skeins and mapping class groups, Math. Proc. Cambridge Phil. Soc., 115 (1994), 53-77.

[41] Y.S. Stanev and I.T. Todorov, On the Schwarz problem for the $\widehat{s u(2)}$ KnizhnikZamolodchikov equation, Lett. Math. Phys., 35 (1995), 123-134. 
[42] A. Tsuchyia and Y. Kanie, Vertex operators in two dimensional conformal field theory on $P^{1}$ and monodromy representations of braid groups, Adv. Studies Pure Math., Tokyo, 16 (1988), 297-372.

[43] A. Tsuchyia, K. Ueno and Y. Yamada, Conformal field theory on universal family of stable curves with gauge symmetries, Adv. Studies Pure Math., Tokyo, 19 (1989), 459-566.

[44] V. Turaev, Quantum invariants of links and 3-valent graphs in 3-manifolds, Publ. Math. I.H.E.S., 77 (1993), 121-171.

[45] _ Quantum invariants of knots and 3-manifolds, Vol. 18. de Gruyter Studies in Math., 1994.

[46] V. Turaev and O.Viro, State-sum invariants of 3-manifolds and quantum $6 j$-symbols, Topology, 31 (1992), 865-902.

[47] V. Turaev and H. Wenzl, Quantum invariants of 3-manifolds associated with classical simple Lie algebras, International Journal of Math., 4 (1993), 323-358.

[48] K. Walker, Chern-Simons-Witten invariants of 3-manifolds, Preprint, 1993.

[49] H. Wenzl, Hecke algebras of type $A_{n}$ and subfactors, Invent. Math., 92 (1988), 349388.

[50] E. Witten, Quantum field theory and the Jones polynomial, Commun. Math. Phys., 121 (1989), 351-399.

[51] G. Wright, The Reshetikhin-Turaev representation of the mapping class groups, J. Knot Theory and Its Ramif., 3 (1994), 547-574.

[52] _ , The Reshetikhin-Turaev representation of the mapping class groups at sixth roots of unity, J. Knot Theory and Its Ramif., 5 (1996), 721-741.

Received June 10, 1997 and revised December 15, 1997.

Institute Fourier, 8P74

Univ. Grenoble I

38402 Saint-Martin-D'Hères CEDEX

FRANCE

E-mail address: funar@fourier.ujf-grenoble.fr 